\title{
Fatores contextuais e implantação da intervenção Housing First: uma revisão da literatura
}

\author{
Contextual factors and implementing the Housing First \\ intervention: a literature review
}

Adriana Pinheiro Carvalho (https://orcid.org/0000-0001-9548-0577) ${ }^{1}$

Juarez Pereira Furtado (https://orcid.org/0000-0001-6605-1925) ${ }^{2}$

${ }^{1}$ Programa de PósGraduação em Saúde Coletiva, Faculdade de Medicina, Universidade de São Paulo. Av. Dr. Arnaldo $4552^{\circ}$ andar sala 2214 , Cerqueira César. 01246-903 São Paulo SP Brasil. adriana.pinheirocarvalho@ gmail.com

${ }^{2}$ Departamento de Políticas Públicas e Saúde Coletiva,

Universidade Federal de São

Paulo. Santos SP Brasil.

\begin{abstract}
Housing First (HF) has spread on the international scene as an evidence-based intervention to overcome homelessness among people with mental health problems. In Brazil, the HF has been adopted as a reference in the development of initiatives geared to people living in the streets who make harmful use of drugs. Studies point to the need for greater understanding of the dynamics of implementation in different settings. Thus, using the literature review method, we analyzed the factors that facilitate and hinder the implementation of HF. Sixty-eight papers published from 2003 to 2020 were selected from the PubMed, Scopus, PsychoINFO, Embase, Lilacs, and Scielo databases. We identified factors in four dimensions: intervention characteristics, implementation context, institutional aspects, and implementation process. The unavailability of housing, the lack of coordination of the services needed by residents, and the resistance of implementing agents to the HF principles are factors that hinder the implementation. In turn, agents with values, attitudes, and skills converging with the model and continuing education appear as facilitators. We point out the need to understand and favor the HF integration process in the existing social protection systems.
\end{abstract}

Key words Housing, Homeless people, Mental health, Implementation science
Resumo O Housing First (HF) tem se difundido no cenário internacional como uma intervenção baseada em evidência para superar a situação de rua entre pessoas com agravos de saúde mental. No Brasil, o HF tem sido adotado como referência no desenvolvimento de iniciativas voltadas para pessoas em situação de rua que fazem uso prejudicial de drogas. Estudos apontam a necessidade de maior compreensão da dinâmica implantação do modelo HF em diferentes cenários. De tal modo, utilizando o método de revisão de literatu$\mathrm{ra}$, analisamos os fatores que facilitam e dificultam a implantação do HF. Foram selecionados 68 artigos, publicados entre 2003 e 2020, nas bases PubMed, Scopus, PsychoINFO, Embase, Lilacs e Scielo. Identificamos fatores em quatro dimensões: características da intervenção, contexto de implantação, aspectos institucionais e processo de implantação. A indisponibilidade de habitações, a falta de coordenação dos serviços necessários aos moradores e a resistência dos agentes implantadores aos princípios do HF são fatores que dificultam a implantação. Por sua vez, agentes com valores, atitudes e competências convergentes ao modelo e educação permanente aparecem como facilitadores. Apontamos a necessidade de se compreender e favorecer o processo de integração do HF aos sistemas de proteção social existentes.

Palavras-chave Moradia, Pessoas em situação de rua, Saúde mental, Ciência da implementação 


\section{Introdução}

A compreensão da moradia como um direito social que desempenha papel fundamental no exercício da cidadania constitui o argumento central para a criação de intervenções do tipo moradia apoiada (supportive housing) ${ }^{1,2}$, como o Housing First ${ }^{3}$. Originalmente desenvolvido nos Estados Unidos em 19924, o Housing First (HF) se constitui como uma proposta que busca enfrentar a situação de rua entre pessoas com agravos de saúde mental por meio da oferta de moradia permanente integrada a serviços de apoio habitacional, clínico e de integração comunitária ${ }^{3}$.

O HF diferencia-se dos serviços tradicionais para pessoas em situação de rua por fornecer acesso à moradia sem o cumprimento de requisitos, tais como a adesão a tratamentos de saúde ou a abstinência do uso de drogas. Como pressuposto fundamental, o modelo assume que a estabilidade e a segurança de uma habitação permanente são decisivas para que outros problemas, tais como o uso prejudicial de drogas, sejam enfren$\operatorname{tados}^{3,5}$. Nesse sentido, embora serviços de apoio sejam oferecidos, esses são disponibilizados de forma independente da moradia e os próprios beneficiários decidem se querem e como vão participar (tipo de acompanhamento, intensidade e frequência).

O HF foi disseminado nos Estados Unidos ${ }^{4}$, Canadá $^{6}$, países europeus ${ }^{7}$ e Austrália ${ }^{8}$ por meio de projetos experimentais, integrados a pesquisas avaliativas, que apontaram a sua maior eficácia quando comparado a intervenções tradicionais de moradia para população em situação de rua. Nesses estudos, o tempo de permanência é o principal indicador de eficácia utilizado, representado pelo percentual de pessoas (que no HF chega a $80 \%$ ), que permanece na moradia por mais de dois anos ${ }^{9}$. Resultados positivos também são relatados com relação à integração comunitária ${ }^{10}$, diminuição do uso de álcool e outras drogas $^{11,12}$ e controle de $\mathrm{HIV}^{13}$. Análises comparativas demonstram ainda que o HF está associado à diminuição de custos provocados pela redução da utilização de outros serviços públicos, como abrigos, hospitais e prisões ${ }^{14}$.

Nos últimos anos, verifica-se o surgimento da discussão sobre o HF em países da América Latina e a implantação de iniciativas inspiradas no modelo no Uruguai ${ }^{15}$ e Chile. No Brasil, o Governo Federal adotou, em 2014, o HF como referência para o desenvolvimento de um conjunto de experiências locais de moradia apoiada específicas para usuários de drogas em situação de rua ${ }^{16-18}$. Atualmente, o modelo tem sido deba- tido no país como uma solução para a falta de moradia para a população em situação de rua em geral ${ }^{19,20} \mathrm{e}$ foi instituído como um programa nacional - Programa Moradia Primeiro - vinculado ao atual Ministério da Mulher, da Família e dos Direitos Humanos ${ }^{21}$.

A rápida expansão do HF resultou em diferentes modificações da proposta original estadunidense, gerando incertezas de como as particularidades locais influenciam a eficácia da intervenção ${ }^{5}$, sendo apontada a necessidade de maior compreensão dos processos de implantação desse tipo de programa em diferentes cenários $^{22}$.

Considera-se que a ruptura com a abordagem "tratamento primeiro", que exige da pessoa beneficiada a demonstração prévia de capacidades consideradas necessárias à estabilidade habitacional, implica em mudanças expressivas no campo das políticas de atenção à população em situação de rua, nos níveis individual, organizacional e político, conferindo complexidade ao HF e ao seu processo de implantação $0^{23,24}$. Tal proposta de transformação requer o envolvimento de diferentes grupos de interesse, a consideração de características locais, além de adaptações significativas nos sistemas de prestação de serviços existentes ${ }^{25}$, ou seja, os chamados fatores contextuais.

No cenário global de expansão do Housing First como uma intervenção promissora no enfrentamento da situação de rua, é fundamental compreender os caminhos pelos quais o modelo vem sendo implantado e as variáveis subjacentes a esse processo. Identifica-se estudo que discute aspectos da implantação do conjunto de projetos experimentais desenvolvidos no Canadá ${ }^{26}$, entretanto não encontramos síntese crítica sobre a influência de fatores contextuais na implantação do modelo HF. Assim, o estudo propôs identificar e discutir os aspectos que facilitam e dificultam a implantação do HF a fim subsidiar tanto o desenvolvimento da proposta original quanto o enfrentamento dos desafios provenientes de sua adaptação a diferentes contextos, incluindo o brasileiro.

\section{Método}

O levantamento de artigos foi realizado em março de 2020, nas bases PubMed, Scopus, PsychoINFO, Embase, Lilacs e Scielo, escolhidas por conterem um expressivo número de periódicos indexados e serem referências na área da saúde. Utilizamos a expressão "housing first" associada simultaneamente às palavras "implementation", 
"translation", "fidelity", "dissemination", "variation" e "application". A busca foi realizada pelo cruzamento dos assuntos, títulos e resumos. Optamos por não realizar um filtro para a data de publicação, para recuperar todos os trabalhos publicados até a data da busca.

A seleção obedeceu aos seguintes critérios de elegibilidade: estudo empírico publicado em periódico científico em qualquer idioma ou ano; intervenção definida como Housing First e destinada para pessoas em situação de rua, independentemente de outros grupos populacionais estarem incluídos; e avaliação ou descrição de fatores relacionados ao processo de implantação de intervenções de HF. Para os propósitos da revisão, foram considerados fatores contextuais os elementos relatados como dificuldades e facilidades ao processo de implantação.

Com o apoio do gerenciador de referências Mendeley, todas as publicações levantadas nas bases de dados foram organizadas e as duplicações removidas. Posteriormente, os artigos foram exportados para o software Rayyan ${ }^{27}$, onde foi realizada a análise dos títulos e resumos e excluídos os artigos que não atenderam os critérios de elegibilidade acima descritos. Os artigos resultantes foram lidos integralmente, excluindo-se os não elegíveis. Por fim, as referências bibliográficas dos artigos selecionados foram verificadas manualmente para inclusão de outros estudos elegíveis anteriormente não identificados. $\mathrm{O}$ processo foi realizado, de forma independente, por dois pesquisadores com experiência prévia no tema da moradia em saúde mental e as discordâncias resolvidas por meio de discussão entre os dois pesquisadores.

A extração de dados dos artigos selecionados foi realizada por uma pesquisadora e verificada por outro pesquisador, ao longo do processo, para garantir precisão. As seguintes informações foram extraídas: dados da publicação (autoria, país de realização do estudo, área temática do periódico e ano de publicação); desenho do estudo (objetivo, método, participantes da pesquisa e referencial teórico); características da intervenção e do contexto (fase de implantação, órgão financiador, política setorial vinculada, organização responsável pela gestão e execução, arranjo institucional, público-alvo, tipo de moradia, serviços de apoio ofertados); e fatores que dificultaram ou facilitaram a implantação.

Os fatores contextuais extraídos dos artigos foram listados e agrupados em quatro dimensões, baseadas no Consolidate Framework for Implementation Research (CFIR) ${ }^{25}$, modelo analítico utilizado no campo da saúde na iden- tificação e compreensão dos fatores contextuais que influenciam o processo de implantação. As semelhanças entre os fatores evidenciados pelos estudos foram também identificadas, agrupadas e descritas. Por fim, os dados foram sintetizados a partir da interpretação da relação existente entre os fatores contextuais e as dimensões do Housing First, narrando como os elementos internos e externos da intervenção influenciam o processo de implantação do modelo.

\section{Resultados}

Foram identificadas 377 publicações, das quais foram selecionados 68 artigos atendendo os critérios de elegibilidade definidos no estudo (Figura 1$)$.

\section{Características dos estudos e intervenções}

Os 68 artigos selecionados abrangem o período entre 2003 e 2020, sendo a maior parte publicada entre 2014 e 2020 (Quadro 1) e em periódicos classificados como da área Saúde, que abarcam 37 publicações, destacando-se a subárea da Saúde Mental com dez artigos. Em seguida, destacam-se os periódicos das áreas de Habitação e Urbanismo, com 11 publicações; das Ciências Sociais e Política, com oito publicações; e de Psicologia Comunitária, com seis publicações. Artigos em menor número (entre um e quatro) encontram-se em revistas das áreas de Serviço Social e Drogas. A ausência de estudos anteriores a 2003 pode ser explicada pela utilização do termo Housing First em publicações científicas ter surgido a partir do ano $2001^{4}$.

A maioria das pesquisas abordou a implantação do HF nos Estados Unidos e no Canadá, totalizando $85 \%$ dos estudos analisados. Os contextos europeu e australiano compõem o restante dos estudos com seis e quatro artigos respectivamente. Predominam dados produzidos por meio de métodos qualitativos (72\%), utilizando grupo focal e entrevistas, sendo três estudos exclusivamente quantitativos ${ }^{28-30}$. A maioria dos estudos teve mais de um grupo de interesse como fonte de informação, sendo trabalhadores e gestores os mais envolvidos $(n=43)$, seguidos por beneficiá$\operatorname{rios}(\mathrm{n}=35)$.

Os artigos que explicitam os desenhos metodológicos indicam abordagens avaliativas $(n=11)$, estudo de caso $(n=15)$, etnografia $(n=4)$ e grounded theory $(n=7)$. Os 18 estudos que informam referencial teórico ou conceitual apontam o Interacionismo simbólico, o Construtivismo ${ }^{31,32}$, 


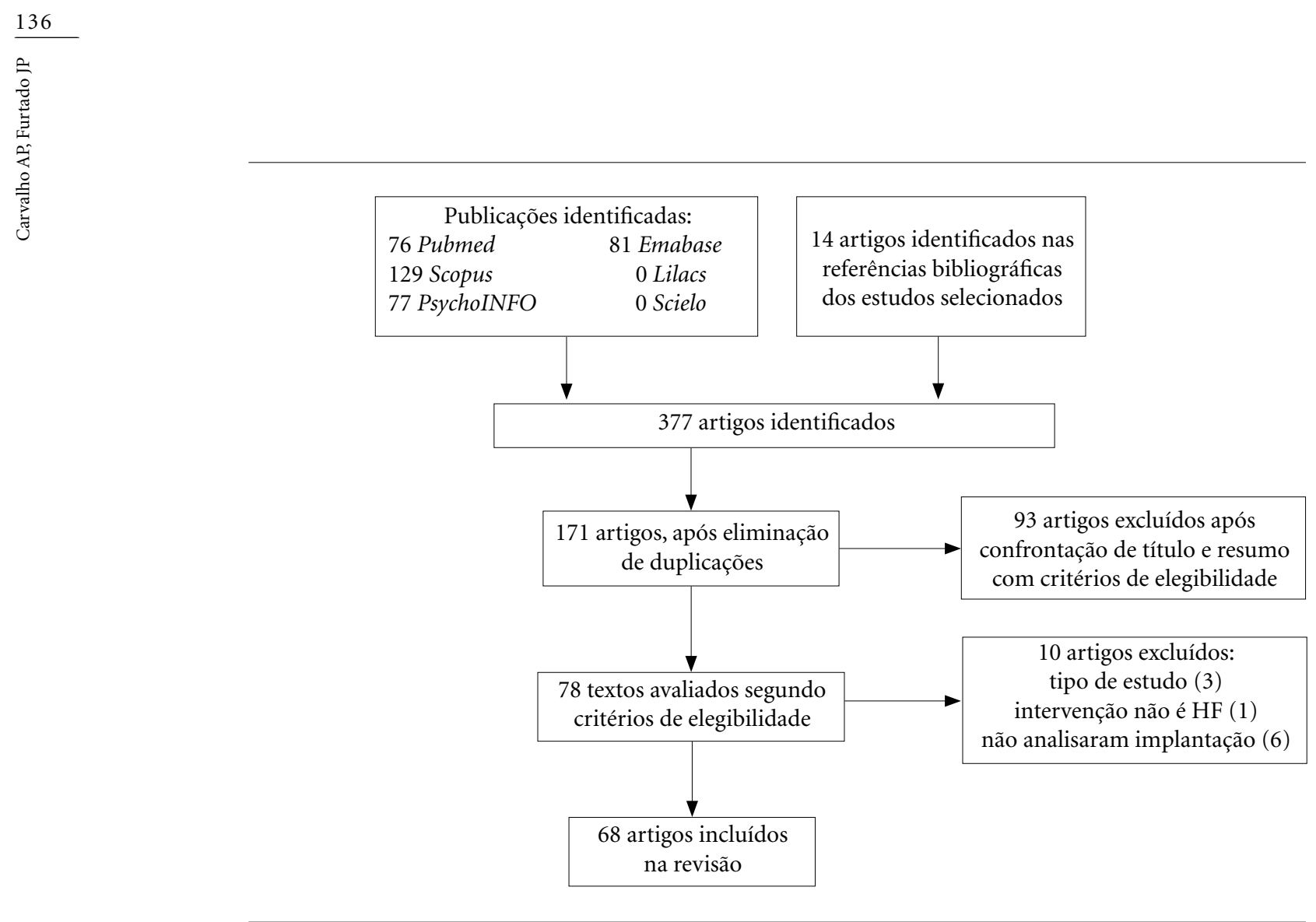

Figura 1. Fluxograma de seleção dos artigos.

Fonte: Autores.

os frameworks oriundos da ciência da implantação ${ }^{30,33-39}$, as teorias da mudança e transformação ${ }^{23,40-43}$ e Street Level Bureaucracy ${ }^{44,45}$.

De maneira geral, a implantação foi abordada com base no binômio estruturação das moradias e oferta de apoio aos moradores, tendo como parâmetros a qualidade das ações executadas e a fidelidade ao modelo original HF. Um conjunto menor de artigos explorou o momento inicial da implantação, especialmente aspectos relacionados ao planejamento e a condições locais para implantação da intervenção. Quatro artigos tiveram como tema central a sustentabilidade das intervenções ${ }^{46-49}$.

Quase um terço ou 21 estudos analisaram intervenções-piloto de HF, sendo 18 oriundas do projeto canadense At Home Chez Soi ${ }^{6}$, maior estudo experimental do HF já desenvolvido ${ }^{12}$, que avaliou os resultados e o processo de implantação do modelo em cinco cidades do país e atendeu aproximadamente 2 mil pessoas em situação de rua com problemas de saúde mental. Ainda no contexto canadense, identificam-se estudos que examinaram intervenções vinculadas a outra iniciativa financiada pelo Governo Federal para ex- pandir o HF - o Homelessness Partnering Strategy $(H P S)^{43}$. Nas pesquisas estadunidenses, prevalecem estudos de programas derivados do projeto Full-Service Partnerships (FSPs) ${ }^{12}$, financiado por fundo de serviço de saúde mental do estado da Califórnia (Mental Health Services Fund $M H S F)$ e da iniciativa firmada entre o setor federal de habitação e urbanismo - US Department of Housing and Urban Development (HUD) e serviços de moradia apoiada para veteranos - VA's Supportive Housing Services (VASH) ${ }^{43}$. O projetopiloto Housing First Europe e a iniciativa Inner City Integrated Services Coalitions ${ }^{43}$ destacam-se dentre os estudos europeus e australianos.

A maior parte dos estudos não informou a natureza jurídica da organização responsável pela intervenção (pública ou privada), a fonte de financiamento e o modelo de gestão adotado. Dentre os que o fizeram, destacam-se: saúde mental e habitação como os setores responsáveis pela intervenção; fontes governamentais federais como principal fonte de financiamento; e organizações não governamentais, tais como as instituições prestadoras dos serviços que compõem as intervenções. 
Quadro 1. Artigos incluídos na revisão.

\begin{tabular}{|c|c|c|c|}
\hline $\begin{array}{c}\text { Aspecto da } \\
\text { implantação }\end{array}$ & Estudo & Autor, Título, Revista & País \\
\hline \multirow[t]{10}{*}{$\begin{array}{l}\text { Execução do } \\
\text { apoio aos } \\
\text { moradores }\end{array}$} & E1 & $\begin{array}{l}\text { Barrenger SL et al. Discursive Processes Creating Team Culture and } \\
\text { Recovery Orientation among Housing First Providers. Am J Psychiatr } \\
\text { Rehabil 2015;18(3):247-64 }\end{array}$ & EUA \\
\hline & E2 & $\begin{array}{l}\text { Andvig ES et al. Harm reduction in a Norwegian housing first project: } \\
\text { a qualitative study of the treatment providers' practice. Adv Dual } \\
\text { Diagn } 2018 ; 11(1): 4-15\end{array}$ & Noruega \\
\hline & E3 & $\begin{array}{l}\text { Tiderington E, Stanhope V, Henwood BF. A qualitative analysis of case } \\
\text { managers'use of harm reduction in practice. J Subst Abuse Treat 2013; } \\
\text { 44(1):71-7 }\end{array}$ & EUA \\
\hline & $\mathrm{E} 4$ & $\begin{array}{l}\text { Watson DP et al. Housing First and harm reduction: A rapid review } \\
\text { and document analysis of the US and Canadian open-access literature. } \\
\text { Harm Reduct J 2017;14(1) }\end{array}$ & EUA \\
\hline & E5 & $\begin{array}{l}\text { Greenberg B et al. Supportive housing best practices in a mid-sized US } \\
\text { urban community. Housing, Care Support. 2013;16(1):6-15 }\end{array}$ & EUA \\
\hline & E6 & $\begin{array}{l}\text { Collins SE et al. Exploring transitions within a project-based housing } \\
\text { first setting: Qualitative evaluation and practice implications. J Health } \\
\text { Care Poor Underserved 2012;23(4):1678-1697 }\end{array}$ & EUA \\
\hline & E7 & $\begin{array}{l}\text { Stanhope V. The ties that bind: Using ethnographic methods to } \\
\text { understand service engagement. Qual Soc Work 2012; 11(4):412-430 }\end{array}$ & EUA \\
\hline & E8 & $\begin{array}{l}\text { van den Berk-Clark C. The Dilemmas of Frontline Staff Working with } \\
\text { the Homeless: Housing First, Discretion, and the Task Environment. } \\
\text { Hous Policy Debate 2016;26(1):105-122 }\end{array}$ & EUA \\
\hline & E9 & $\begin{array}{l}\text { Brothers S et al. Food insecurity among formerly homeless youth } \\
\text { in supportive housing: A social-ecological analysis of a structural } \\
\text { intervention. Soc Sci Med 2020; 245:112724 }\end{array}$ & EUA \\
\hline & E10 & $\begin{array}{l}\text { Worton SK. Examining peer networking as a capacity building } \\
\text { strategy for Housing First implementation. J Community Psychol 2020; } \\
\text { 48(4):1147-1162 }\end{array}$ & Canadá \\
\hline \multirow{6}{*}{$\begin{array}{l}\text { Adaptação } \\
\text { do HF a um } \\
\text { contexto } \\
\text { específico }\end{array}$} & E11 & $\begin{array}{l}\text { Ecker J et al. Implementation evaluation of a housing first program in } \\
\text { a small Canadian City. Can J Community Ment Heal 2014; 33(4):23-40 }\end{array}$ & Canadá \\
\hline & E12 & $\begin{array}{l}\text { Nolin D, Jetté J. Specific features of urban and rural areas: A } \\
\text { comparative study on the results of the At Home/Chez Soi project in } \\
\text { New Brunswick. Can J Community Ment Heal 2015; 33(4):125-140 }\end{array}$ & Canadá \\
\hline & E13 & $\begin{array}{l}\text { Stefancic A et al. Implementing Housing First in rural areas: Pathways } \\
\text { Vermont. Am J Public Health 2013; 103(Supl. 2):S206-209 }\end{array}$ & EUA \\
\hline & E14 & $\begin{array}{l}\text { Jetté J et al. The implementation of a Housing First intervention in } \\
\text { Canadian rural region Can. J Community Ment Heal 2015; 33(4):41-59 }\end{array}$ & Canadá \\
\hline & E15 & $\begin{array}{l}\text { Austin EL et al. VA's expansion of supportive housing: Successes and } \\
\text { challenges on the path toward housing first. Psychiatr Serv 2014; } \\
\text { 65(5):641-647 }\end{array}$ & EUA \\
\hline & E16 & $\begin{array}{l}\text { Kriegel LS et al. Implementation and Outcomes of Forensic Housing } \\
\text { First Programs. Community Ment Health J 2016; 52(1):46-55 }\end{array}$ & EUA \\
\hline \multirow{3}{*}{$\begin{array}{l}\text { Aspectos gerais } \\
\text { do processo de } \\
\text { implantação }\end{array}$} & E17 & $\begin{array}{l}\text { De Ara CGG et al. Housing first: At home recovery of severe mental } \\
\text { disorder. Rev Med Suisse 2017; 13(575):1605-1609 }\end{array}$ & Suíça \\
\hline & E18 & $\begin{array}{l}\text { Fleury M-J, Grenier G, Vallée C. Evaluation of the implementation of } \\
\text { the Montreal at home/chez soi project. BMC Health Serv Res 2014; } \\
14(1)\end{array}$ & Canadá \\
\hline & E19 & $\begin{array}{l}\text { Benjaminsen L. Policy Review Up-date: Results from the Housing } \\
\text { First based Danish Homelessness Strategy. Eur J Homelessness 2013; } \\
\text { 7(2):109-31 }\end{array}$ & Dinamarca \\
\hline
\end{tabular}


Quadro 1. Artigos incluídos na revisão.

\begin{tabular}{|c|c|c|c|}
\hline $\begin{array}{l}\text { Aspecto da } \\
\text { implantação }\end{array}$ & Estudo & Autor, Título, Revista & País \\
\hline \multirow[t]{4}{*}{$\begin{array}{l}\text { Aspectos } \\
\text { institucionais }\end{array}$} & E20 & $\begin{array}{l}\text { Nicholls CM, Atherton I. Housing First: Considering components for } \\
\text { successful resettlement of homeless people with multiple needs. Hous } \\
\text { Stud 2011; 26(5):767-777 }\end{array}$ & EUA \\
\hline & E21 & $\begin{array}{l}\text { Weinzierl C, Wukovitsch F, Novy A. Housing First in Vienna: a socially } \\
\text { innovative initiative to foster social cohesion. J Hous Built Environ } \\
\text { 2016; 31(3):409-422 }\end{array}$ & Áustria \\
\hline & E22 & $\begin{array}{l}\text { Kertesz SG et al. Making Housing First Happen: Organizational } \\
\text { Leadership in VA’s Expansion of Permanent Supportive Housing. J Gen } \\
\text { Intern Med 2014; 29(4):835-44 }\end{array}$ & EUA \\
\hline & E23 & $\begin{array}{l}\text { Nelson G, Worton SK, Macnaughton E, Tsemberis S, MacLeod T, } \\
\text { Hasford J, et al. Systems change in the context of an initiative to scale } \\
\text { up Housing First in Canada. J Community Psychol 2019; 47(1):7-20. }\end{array}$ & Canadá \\
\hline \multirow{13}{*}{$\begin{array}{l}\text { Avaliação ou } \\
\text { análise da } \\
\text { intervenção } \\
\text { utilizando como } \\
\text { parâmetro a } \\
\text { fidelidade ao } \\
\text { modelo HF }\end{array}$} & E24 & $\begin{array}{l}\text { Gilmer TP et al. Variation in the implementation of California's full } \\
\text { service partnerships for persons with serious mental illness. Health } \\
\text { Serv Res 2013; 48:2245-2267 }\end{array}$ & EUA \\
\hline & E25 & $\begin{array}{l}\text { Greenwood RM et al. Implementations of housing first in Europe: } \\
\text { Successes and challenges in maintaining model fidelity. Am J Psychiatr } \\
\text { Rehabil 2013; 16(4):290-312 }\end{array}$ & Europa \\
\hline & E26 & $\begin{array}{l}\text { Kertesz SG et al. Housing first on a large scale: Fidelity strengths } \\
\text { and challenges in the VA's HUD-VASH program. Psychol Serv 2017; } \\
14(2): 118-128\end{array}$ & EUA \\
\hline & E27 & $\begin{array}{l}\text { O'Campo P et al. Strategies to balance fidelity to Housing First } \\
\text { principles with local realities: lessons from a large urban centre. J } \\
\text { Health Care Poor Underserved 2015; 26(2):536-553 }\end{array}$ & Canadá \\
\hline & E28 & $\begin{array}{l}\text { Macnaughton E et al. Implementing Housing First Across Sites and } \\
\text { Over Time: Later Fidelity and Implementation Evaluation of a Pan- } \\
\text { Canadian Multi-site Housing First Program for Homeless People with } \\
\text { Mental Illness. Am J Community Psychol 2015; 55(3-4):279-291 }\end{array}$ & Canadá \\
\hline & E29 & $\begin{array}{l}\text { Nelson G et al. Early implementation evaluation of a multi-site } \\
\text { housing first intervention for homeless people with mental illness: A } \\
\text { mixed methods approach. Eval Program Plann 2014; 43:16-26 }\end{array}$ & Canadá \\
\hline & E30 & $\begin{array}{l}\text { Stergiopoulos V et al. Moving from rhetoric to reality: Adapting } \\
\text { Housing First for homeless individuals with mental illness from } \\
\text { ethno-racial groups. BMC Health Serv Res 2012;12(1) }\end{array}$ & Canadá \\
\hline & E31 & $\begin{array}{l}\text { Gilmer TP et al. Fidelity to the housing first model and variation in } \\
\text { health service use within permanent supportive housing. Psychiatr } \\
\text { Serv 2015; 66(12):1283-1289 }\end{array}$ & EUA \\
\hline & E32 & $\begin{array}{l}\text { Gilmer TP et al. Fidelity to the housing first model and effectiveness of } \\
\text { permanent supported housing programs in California. Psychiatr Serv } \\
\text { 2014; 65(11):1311-1317 }\end{array}$ & EUA \\
\hline & E33 & $\begin{array}{l}\text { Macnaughton E et al. Navigating complex implementation contexts: } \\
\text { Overcoming barriers and achieving outcomes in a national initiative } \\
\text { to scale out housing first in Canada. Am J Community Psychol 2018; } \\
62(1-2): 135-149\end{array}$ & Canadá \\
\hline & E34 & $\begin{array}{l}\text { Gilmer TP et al. Variations in full service partnerships and fidelity to } \\
\text { the housing first model. Am J Psychiatr Rehabil 2013;16(4):313-328 }\end{array}$ & EUA \\
\hline & E35 & $\begin{array}{l}\text { Watson DP et al. The Housing First Model (HFM) fidelity index: } \\
\text { designing and testing a tool for measuring integrity of housing } \\
\text { programs that serve active substance users. Subst Abus Treat Prev } \\
\text { Policy. 2013; 8(1):16 }\end{array}$ & EUA \\
\hline & E36 & $\begin{array}{l}\text { Fenwick K et al. Exploring Variation in Housing First Implementation: } \\
\text { The Role of Fit. Hum Serv Organ Manag Leadersh Gov 2019; } \\
\text { 43(5):392-406 }\end{array}$ & EUA \\
\hline
\end{tabular}


Quadro 1. Artigos incluídos na revisão.

\begin{tabular}{|c|c|c|c|}
\hline $\begin{array}{c}\text { Aspecto da } \\
\text { implantação }\end{array}$ & Estudo & Autor, Título, Revista & País \\
\hline \multirow[t]{10}{*}{$\begin{array}{l}\text { Processo de } \\
\text { estruturação das } \\
\text { moradias }\end{array}$} & E37 & $\begin{array}{l}\text { Dickson-Gomez J et al. Identifying variability in permanent supportive } \\
\text { housing: A comparative effectiveness approach to measuring health } \\
\text { outcomes. Am J Orthopsychiatry } 2017 ; 87(4): 414-424\end{array}$ & EUA \\
\hline & E38 & Chen PM. Housing first and single-site housing. Soc Sci 2019; 8(4) & EUA \\
\hline & E39 & $\begin{array}{l}\text { Zerger S et al. Understanding Housing Delays and Relocations Within } \\
\text { the Housing First Model. J Behav Health Serv Res 2016; 43(1):38-53 }\end{array}$ & Canadá \\
\hline & E40 & $\begin{array}{l}\text { Pankratz C, Nelson G, Morrison M. The implementation of a } \\
\text { rent assistance program and its impacts on recovery outcomes for } \\
\text { individuals experiencing chronic homelessness. Can J Community } \\
\text { Ment Heal 2018;37(1):49-63 }\end{array}$ & Canadá \\
\hline & E41 & $\begin{array}{l}\text { Henwood BF, Stanhope V, Padgett DK. The role of housing: a } \\
\text { comparison of front-line provider views in housing first and } \\
\text { traditional programs. Adm Policy Ment Heal Ment Heal Serv Res 2011; } \\
38(2): 77-85\end{array}$ & EUA \\
\hline & E42 & $\begin{array}{l}\text { Colombo F, Saruis T. Social innovation and local welfare: A } \\
\text { comparative case study on housing first in Italy and Sweden. } \\
\text { Vol. 33, Journal fur Entwicklungspolitik. Mattersburger Kreis fur } \\
\text { Entwicklungspolitik; 2017.p. 85-111 }\end{array}$ & Itália \\
\hline & E43 & $\begin{array}{l}\text { Kertesz SG et al. The role and meaning of interim housing in housing } \\
\text { first programs for people experiencing homelessness and mental } \\
\text { illness. Am J Orthopsychiatry 2014;84(4):431-437 }\end{array}$ & Canadá \\
\hline & E44 & $\begin{array}{l}\text { Verdouw J, Habibis D. Housing First programs in congregate-site } \\
\text { facilities: can one size fit all? Hous Stud 2018;33(3):386-407 }\end{array}$ & Austrália \\
\hline & E45 & $\begin{array}{l}\text { Bullen J, Baldry E. 'I waited } 12 \text { months': how does a lack of access to } \\
\text { housing undermine Housing First? Int J Hous Policy 2019; 19(1):120- } \\
130\end{array}$ & Austrália \\
\hline & E46 & $\begin{array}{l}\text { Bullen J, Fisher KR. Is Housing First for Mental Health Community } \\
\text { Support Possible During a Housing Shortage? Soc Policy Adm 2015; } \\
\text { 49(7):928-945 }\end{array}$ & Austrália \\
\hline \multirow{6}{*}{$\begin{array}{l}\text { Fatores } \\
\text { relacionados } \\
\text { à pré- } \\
\text { implantação } \\
\text { (planejamento, } \\
\text { construção } \\
\text { da agenda, } \\
\text { formação } \\
\text { permanente) }\end{array}$} & E47 & $\begin{array}{l}\text { Hasford J et al. Knowledge translation and implementation of housing } \\
\text { first in Canada: A qualitative assessment of capacity building needs for } \\
\text { an evidence-based program. Eval Program Plann 2019; 75:1-9 }\end{array}$ & Canadá \\
\hline & E48 & $\begin{array}{l}\text { Worton SK et al. Understanding Systems Change in Early } \\
\text { Implementation of Housing First in Canadian Communities: An } \\
\text { Examination of Facilitators/Barriers, Training/Technical Assistance, } \\
\text { and Points of Leverage. Am J Community Psychol 2018; 61(1-2):118- } \\
130\end{array}$ & Canadá \\
\hline & E49 & $\begin{array}{l}\text { Nelson G et al. Collaboration and involvement of persons with lived } \\
\text { experience in planning Canada's At Home/Chez Soi project. Health Soc } \\
\text { Care Community 2016; 24(2):184-193 }\end{array}$ & Canadá \\
\hline & E50 & $\begin{array}{l}\text { Macnaughton E, Nelson G, Goering P. Bringing politics and evidence } \\
\text { together: Policy entrepreneurship and the conception of the At Home/ } \\
\text { Chez Soi Housing First Initiative for addressing homelessness and } \\
\text { mental illness in Canada. Soc Sci Med 2013; 82:100-107 }\end{array}$ & Canadá \\
\hline & E51 & $\begin{array}{l}\text { Nelson G et al. Planning a Multi-site, Complex Intervention for } \\
\text { Homeless People with Mental Illness: The Relationships Between the } \\
\text { National Team and Local Sites in Canada's At Home/Chez Soi Project. } \\
\text { Am J Community Psychol 2013; 51(3-4):347-358 }\end{array}$ & Canadá \\
\hline & E52 & $\begin{array}{l}\text { Keller C et al. Initial implementation of housing first in five Canadian } \\
\text { cities: How do you make the shoe fit, when one size does not fit all? } \\
\text { Am J Psychiatr Rehabil 2013; 16(4):275-289 }\end{array}$ & Canadá \\
\hline
\end{tabular}


Quadro 1. Artigos incluídos na revisão.

\begin{tabular}{|c|c|c|c|}
\hline $\begin{array}{l}\text { Aspecto da } \\
\text { implantação }\end{array}$ & Estudo & Autor, Título, Revista & País \\
\hline \multirow[t]{3}{*}{$\begin{array}{l}\text { Influência da } \\
\text { implantação } \\
\text { nos resultados } \\
\text { do programa }\end{array}$} & E53 & $\begin{array}{l}\text { Aubry T, Nelson G, Tsemberis S. Housing first for people with severe } \\
\text { mental illness who are homeless: A review of the research and findings } \\
\text { from the at Home-Chez soi demonstration project. Can J Psychiatry } \\
2015 ; 60(11): 467-474\end{array}$ & Canadá \\
\hline & E54 & $\begin{array}{l}\text { Davidson C et al. Association of housing first implementation and key } \\
\text { outcomes among homeless persons with problematic substance use. } \\
\text { Psychiatr Serv 2014; 65(11):1318-1324 }\end{array}$ & EUA \\
\hline & E55 & $\begin{array}{l}\text { Appel PW, Tsemberis S, Joseph H, Stefancic A, Lambert-Wacey D, P.W. } \\
\text { A, et al. Housing first for severely mentally ill homeless methadone } \\
\text { patients. J Addict Dis 2012; 31(3):270-277 }\end{array}$ & EUA \\
\hline \multirow{7}{*}{$\begin{array}{l}\text { Percepção } \\
\text { dos grupos de } \\
\text { interesse sobre } \\
\text { a implantação } \\
\text { do HF }\end{array}$} & E56 & $\begin{array}{l}\text { Henwood BF et al. Examining provider perspectives within housing } \\
\text { first and traditional programs. Am J Psychiatr Rehabil. 2013; } \\
\text { 16(4):262-274 }\end{array}$ & EUA \\
\hline & E57 & $\begin{array}{l}\text { Volk JS et al. The at home / Chez Soi project: Community partners' } \\
\text { perspectives on the implementation of housing first in Moncton. Can J } \\
\text { Community Ment Heal 2015;33(4):77-90 }\end{array}$ & Canadá \\
\hline & E58 & $\begin{array}{l}\text { Felton BJ. Innovation and Implementation in Mental Health Services } \\
\text { for Homeless Adults: A Case Study. Community Ment Health J 2003; } \\
\text { 39(4):309-322. }\end{array}$ & EUA \\
\hline & E59 & $\begin{array}{l}\text { Johnsen S, Teixeira L. “Doing it Already?": Stakeholder Perceptions of } \\
\text { Housing First in the UK. Int J Hous Policy 2012; 12(2):183-203. }\end{array}$ & $\begin{array}{l}\text { Reino } \\
\text { Unido }\end{array}$ \\
\hline & E60 & $\begin{array}{l}\text { Macnaughton EL, Goering PN, Nelson GB. Exploring the value of } \\
\text { mixed methods within the at home/chez Soi housing first project: } \\
\text { A strategy to evaluate the implementation of a complex population } \\
\text { health intervention for people with mental illness who have been } \\
\text { homeless. Can J Public Heal 2012; 103:S57-S62 }\end{array}$ & Canadá \\
\hline & E61 & $\begin{array}{l}\text { Kennedy J, Arku G, Cleave E. The experiences of front-line service } \\
\text { providers of Housing First programme delivery in three communities } \\
\text { in Ontario, Canada. Int J Hous Policy 2017; 17(3):396-416 }\end{array}$ & Canadá \\
\hline & E62 & $\begin{array}{l}\text { Kennedy J et al. Service providers' perspectives and residents' } \\
\text { experiences with the implementation of "Housing First" program in } \\
\text { the city of London, Ontario, Canada. Hous Soc 2016; 43(2):82-102 }\end{array}$ & Canadá \\
\hline \multirow{3}{*}{$\begin{array}{l}\text { Processo de } \\
\text { elegibilidade } \\
\text { dos } \\
\text { participantes }\end{array}$} & E63 & $\begin{array}{l}\text { Osborne M. Who Gets "Housing First"? Determining Eligibility in } \\
\text { an Era of Housing First Homelessness. J Contemp Ethnogr 2019; } \\
\text { 48(3):402-428 }\end{array}$ & EUA \\
\hline & E64 & $\begin{array}{l}\text { Anderson-Baron JT, Collins D. Not a "forever model": the curious case } \\
\text { of graduation in Housing First. Urban Geogr 2018; 39(4):587-605 }\end{array}$ & Canadá \\
\hline & E65 & $\begin{array}{l}\text { Namian D. Governing homelessness through instruments: a critical } \\
\text { perspective on housing first's policy instrumentation. Crit Policy Stud } \\
2020 \text { Jul 2; 14(3):303-318 }\end{array}$ & Canadá \\
\hline \multirow[t]{3}{*}{ Sustentabilidade } & E66 & $\begin{array}{l}\text { Kumar N et al. Sustaining housing first after a successful research } \\
\text { demonstration trial: Lessons learned in a large urban center. Psychiatr } \\
\text { Serv 2017; 68(7):739-42 }\end{array}$ & Canadá \\
\hline & E67 & $\begin{array}{l}\text { Nelson G et al. What Happens After the Demonstration Phase? The } \\
\text { Sustainability of Canada's At Home/Chez Soi Housing First Programs } \\
\text { for Homeless Persons with Mental Illness. Am J Community Psychol } \\
\text { 2017; 59(1-2):144-157 }\end{array}$ & Canadá \\
\hline & E68 & $\begin{array}{l}\text { Stergiopoulos V et al. Dynamic Sustainability: Practitioners' } \\
\text { Perspectives on Housing First Implementation Challenges and Model } \\
\text { Fidelity Over Time. Res Soc Work Pract 2016; 26(1):61-68 }\end{array}$ & Canadá \\
\hline
\end{tabular}


Os estudos abordaram essencialmente programas de HF implantados em áreas urbanas e três pesquisas incluíram iniciativas desenvolvidas no meio rural ${ }^{50-52}$. Dos artigos que especificaram as características dos beneficiários das intervenções, pessoas em situação de rua com agravos de saúde mental e/ou uso de drogas foi o público -alvo predominante. Alguns programas atenderam pessoas com vivência de rua em situações específicas: pessoas jovens ${ }^{53}$, pessoas negras com agravos de saúde mental ${ }^{54}$, mulheres com filhos ${ }^{42}$, pessoas atendidas em serviços do sistema penal ${ }^{55}$ e veteranas de guerra ${ }^{40,41}$.

O tipo de moradia e de serviços de apoio ofertados pelos programas não é especificado em todos os estudos. Identificam-se programas que utilizaram apartamentos individuais dispersos, unidades congregadas nas quais os participantes moravam com outros beneficiários em um mesmo edifício e finalmente aqueles que adotaram as duas opções de moradia.

Os serviços de apoio são predominantemente ofertados por meio de Case Management (Gerenciamento de Casos) ou integrando o Gerenciamento de Casos e o ACT (Assertive Community Treatment ou Tratamento Assertivo na Comunidade). Ambos constituem modelos de cuidado de base comunitária indicados pelo HF como proposta de operacionalização do apoio a ser ofertado aos moradores. O ACT é mais frequentemente empregado para pessoas com grande necessidade de apoio em saúde mental e o Gerenciamento de Casos, para aquelas com necessidades moderadas ${ }^{56}$. Os estudos que abordaram programas que utilizaram somente o ACT aparecem com menor destaque ${ }^{29,47,50,57-59}$.

\section{Fatores que influenciam a implantação do $\mathrm{HF}$}

As dificuldades e facilidades enfrentadas no processo de implantação do HF são aqui analisadas com base em quatro dimensões: características da proposta, contexto no qual a intervenção foi introduzida (beneficiários, rede de serviços locais e comunidade), aspectos institucionais (valores, atitudes, competências, recursos e coordenação das ações do programa) e processo de execução da proposta (seleção dos participantes, estruturação das moradias e oferta de apoio aos beneficiários). No Quadro 2, os fatores estão agrupados conforme representem barreiras ou facilitadores e as respectivas fontes indicadas segundo a numeração dos estudos estabelecida no Quadro 1.

\section{Discussão}

A transposição de uma proposta de intervenção para situações concretas encontra apoios e resistências no contexto local ${ }^{60,61}$. No caso específico do Housing First, verifica-se que o conjunto de evidências disponíveis sobre a efetividade da intervenção confere legitimidade ao modelo, favorecendo sua percepção como uma solução mais efetiva do que outras intervenções no enfrentamento do problema da situação de rua ${ }^{62}$. Entretanto, sendo a proposta concebida externamente, os agentes implementadores podem perceber que as capacidades locais não são devidamente consideradas no planejamento e na execução da intervenção e que o modelo traz pouca permeabilidade para adaptações ${ }^{34,62}$.

Ainda com relação às características da proposta que desafiam a implantação, o modelo HF pode ser percebido como complexo, em decorrência do grau de mudança de atitude exigido dos implantadores, da necessidade de interação entre agentes e instituições com diferentes culturas e práticas institucionais, além da falta de clareza operacional do programa. É de notar também que, quando a oportunidade de implantação acontece por meio de projetos-piloto, a incerteza sobre a sustentabilidade da intervenção, após o período demonstrativo, parece também influenciar a falta de engajamento ${ }^{62,63}$.

Atitudes que desviam fundamentos estabelecidos no $\mathrm{HF}^{35,46,64,65}$, especialmente no que diz respeito à focalização do programa em pessoas com necessidades mais complexas (tais como as que fazem uso intenso de drogas e possuem baixa adesão a tratamentos de saúde), comprometem de forma crítica a implantação do modelo. Por exemplo, o estabelecimento de critérios e limite de tempo para a permanência dos participantes no programa $^{44,66,67}$ ameaça o pressuposto de estabilidade habitacional do modelo e diminui a capacidade de interrupção da lógica da moradia temporária, marcada pela alternância entre a vida nas ruas, instituições públicas e habitações precárias.

Essas e outras práticas que contariam os pressupostos do modelo, podem estar associadas a processos mais amplos de reprodução de desigualdades sociais ${ }^{68,69}$, o que pode representar um maior desafio quando a implantação se desenvolve em cenários de relações sociais tão desiguais, tal como no caso brasileiro ${ }^{70}$. Esse debate vem sendo abordado na literatura de políticas públicas sobre discricionariedade, incluindo estudo brasileiro no campo do cuidado relacionado ao uso de drogas ${ }^{71}$. 
Quadro 2. Fatores que influenciam a implantação do HF sistematizados a partir dos estudos referenciados no Quadro 1.

\begin{tabular}{|c|c|c|}
\hline Fatores & Dificultadores & Facilitadores \\
\hline \multicolumn{3}{|c|}{ Características da intervenção } \\
\hline Origem & $\begin{array}{l}\text { - Formulação do modelo por agentes externos (E18, } \\
\text { E49,E58,E51) } \\
\text { - Especificidade da área de surgimento da } \\
\text { intervenção (saúde mental) (E18) }\end{array}$ & \\
\hline Evidências & $\begin{array}{l}\text { - Evidências insuficientes sobre a efetividade para } \\
\text { pessoas que usam drogas (E59) } \\
\text { - Percepção de que o modelo adota estratégias já } \\
\text { utilizadas (E59) }\end{array}$ & $\begin{array}{l}\text { - Percepção do HF como uma solução mais } \\
\text { efetiva do que outros modelos na resolução da } \\
\text { situação de rua (E58) } \\
\text { - Robustez das evidências sobre efetividade do } \\
\text { HF (E49) }\end{array}$ \\
\hline Lógica do modelo & \begin{tabular}{|l} 
- Baixa permeabilidade do modelo ao contexto \\
(E18,E62) \\
- Indefinição conceitual e operacional sobre a \\
Redução de Danos no modelo HF (E63,64) \\
- Pouca clareza sobre a operacionalização do \\
acompanhamento na moradia (64) \\
- Modelo exige elevado grau de interação com \\
organizações e agentes de diferentes setores \\
(E18,E47) \\
- Modelo pressupõe mudança de paradigma, valores \\
e atitudes (E18,E47)
\end{tabular} & \\
\hline \multicolumn{3}{|c|}{ Contexto de implantação } \\
\hline \multicolumn{3}{|c|}{ Características dos Beneficiários } \\
\hline $\begin{array}{l}\text { Atitudes e } \\
\text { costumes }\end{array}$ & \begin{tabular}{|l} 
- Uso intenso de drogas (E20,E28,E41,E40) \\
- Inadimplência com o aluguel (E18) \\
- Conflito com locador e profissional de apoio (E62) \\
- Não comparecimento aos encontros firmados com \\
equipe de acompanhamento (E2,E18) \\
- Percepção negativa sobre experiências em serviços \\
anteriores (E61) \\
- Permanência de convidados não autorizados na \\
moradia (E38,E40) \\
- Resistência em utilizar serviços públicos de \\
alimentação e falta de habilidade para preparo de \\
alimentos (E9) \\
- Diversidade cultural e linguística dos moradores \\
(E30)
\end{tabular} & \\
\hline $\begin{array}{l}\text { Necessidades e } \\
\text { recursos }\end{array}$ & \begin{tabular}{|l} 
- Dificuldade de atendimento das necessidades \\
de saúde, trabalho e educação dos moradores \\
(E3,E27,E29,E61,E62) \\
- Falta de flexibilidade para acomodação de famílias \\
$(61)$ \\
- Isolamento e solidão (E18,E28,E37,E38,E60) \\
- Ausência de renda mínima (E9,E18,E42,E57,E62) \\
- Falta de transporte público (E29) \\
- Pouca oportunidade de inserção no mercado de \\
trabalho (E14) \\
- Pouco envolvimento dos participantes na avaliação \\
do programa (E11,E68)
\end{tabular} & $\begin{array}{l}\text { - Acesso a computador e internet (E13) } \\
\text { - Participação dos moradores no planejamento } \\
\text { dos serviços (E54) }\end{array}$ \\
\hline
\end{tabular}


Quadro 2. Fatores que influenciam a implantação do HF sistematizados a partir dos estudos referenciados no Quadro 1.

\begin{tabular}{|c|c|c|}
\hline Fatores & Dificultadores & Facilitadores \\
\hline \multicolumn{3}{|c|}{ Rede de serviços e comunidade } \\
\hline Abertura à proposta & $\begin{array}{l}\text { - Instituições pouco permeáveis ao novo modelo (E1 } \\
\text { 6,E17,E18,E19,E20,E25,E26,E42,E47,E51,E56,E58) } \\
\text { - Falta de engajamentos das autoridades } \\
\text { governamentais locais (E18,E20) } \\
\text { - Percepção de que a nova intervenção prejudica os } \\
\text { serviços tradicionais existentes (E58,45) } \\
\text { - Agentes de serviços tradicionais locais sentem-se } \\
\text { desqualificados (E48,E58) }\end{array}$ & $\begin{array}{l}\text { - Agentes políticos com credibilidade ajudam na } \\
\text { construção da agenda de implantação (E50) }\end{array}$ \\
\hline Estrutura & $\begin{array}{l}\text { - Baixo número de serviços disponíveis para atender } \\
\text { as necessidades dos moradores (E25,E28,E37,E62) }\end{array}$ & $\begin{array}{l}\text { - Existência de rede de serviços de apoio } \\
\text { comunitários complementares à intervenção } \\
(\mathrm{E} 9, \mathrm{E} 29)\end{array}$ \\
\hline Estigma & $\begin{array}{l}\text { - Atitudes de intolerância, estigma, preconceito e } \\
\text { discriminação por parte dos proprietários e vizinhos } \\
(\mathrm{E} 12, \mathrm{E} 14, \mathrm{E} 20, \mathrm{E} 29, \mathrm{E} 38, \mathrm{E} 57, \mathrm{E} 60)\end{array}$ & \\
\hline \multicolumn{3}{|c|}{ Aspectos institucionais } \\
\hline $\begin{array}{l}\text { Inserção da } \\
\text { instituição } \\
\text { responsável }\end{array}$ & $\begin{array}{l}\text { - Instituição externa à rede local (E58) } \\
\text { - Instituição externa ao campo da saúde mental } \\
\text { (E18) } \\
\text { - Falta de experiência na integração de pessoas } \\
\text { que estiveram em situação de rua (pares) como } \\
\text { membros da equipe (E49) } \\
\text { - Cultura organizacional não alinhada com a } \\
\text { abordagem de Redução de Danos (E48) }\end{array}$ & $\begin{array}{l}\text { - Experiência prévia com serviços de moradia } \\
\text { para população em situação de rua (E30) } \\
\text { - Envolvimento na defesa dos direitos do público } \\
\text { atendido (advocacy) (E62) }\end{array}$ \\
\hline $\begin{array}{l}\text { Valores, crenças } \\
\text { e atitudes dos } \\
\text { gestores e equipe }\end{array}$ & $\begin{array}{l}\text { - Dificuldade em romper com a lógica do } \\
\text { tratamento primeiro e adotar a abordagem de } \\
\text { Redução de Danos (E4,E17,E19,E42,E61,E62) } \\
\text { · Julgamento moral acerca do merecimento da } \\
\text { moradia (E25) } \\
\text { - Falta de adesão da equipe a escolha de moradia do } \\
\text { participante (E43) } \\
\text { - Atitudes, autoritárias, estigmatizantes e hostis } \\
(\text { E1,E24,E26) } \\
\text { - Ambiguidade com relação ao tempo de } \\
\text { permanência dos beneficiários no programa (E64) } \\
\text { - Prioridades e expectativas conflitantes entre } \\
\text { profissionais do serviço de habitação e gerentes de } \\
\text { caso (E39) }\end{array}$ & $\begin{array}{l}\text { - Equipe e gestores com valores, habilidades } \\
\text { e atitudes alinhadas aos objetivos do modelo } \\
\text { (E24,E29,E34, E49) } \\
\text { - Atitudes positivas da gestão de alto escalão } \\
\text { (alocação de recursos, monitoramento de } \\
\text { performance e participação dos gestores de } \\
\text { médio escalão) (E15) } \\
\text { - Liderança do gestor local (habilidade de } \\
\text { tomada de decisão, comunicação clara e direta, } \\
\text { promoção de clima cooperativo) (E29) }\end{array}$ \\
\hline $\begin{array}{l}\text { Capacidades e } \\
\text { competências dos } \\
\text { gestores e equipe }\end{array}$ & $\begin{array}{l}\text { - Falta de compreensão dos princípios do HF (E48) } \\
\text { - Equipe sem qualificação e repertório prático de } \\
\text { competências consideradas essenciais à implantação } \\
\text { (redução de danos, entrevista motivacional, } \\
\text { planejamento centrado no usuário, abordagem } \\
\text { racial (E4,E6,E27,E29,E30,E33,E35,E36,E57) }\end{array}$ & $\begin{array}{l}\text { - Clareza sobre o modelo HF (E20) } \\
\text { - Habilidade e autonomia para realizar } \\
\text { julgamentos clínicos (E3) } \\
\text { - Capacidade de manter bom relacionamento } \\
\text { com os locadores (E60) } \\
\text { - Equipe com experiência prévia em serviços de } \\
\text { moradia assistida (E24,E29,E34) } \\
\text { - Boa comunicação com pessoas com transtorno } \\
\text { mental grave (55) } \\
\text { - Equipe com capacidade de integrar o cuidado } \\
\text { em álcool e drogas ao apoio de saúde mental } \\
(17)\end{array}$ \\
\hline $\begin{array}{l}\text { Educação } \\
\text { permanente }\end{array}$ & $\begin{array}{l}\text { - Falta de supervisão técnica para equipe (E18) } \\
\text { - Falta de treinamento para enfrentamento de } \\
\text { problemas habitacionais (mudança de casa e } \\
\text { realocação) e de agravos de saúde mental (E26,E29) }\end{array}$ & $\begin{array}{l}\text { - Treinamento e supervisão técnica permanente } \\
\text { conduzido por pessoas experientes no modelo } \\
\text { HF (E52) }\end{array}$ \\
\hline
\end{tabular}


Quadro 2. Fatores que influenciam a implantação do HF sistematizados a partir dos estudos referenciados no Quadro 1.

\begin{tabular}{|c|c|c|}
\hline Fatores & Dificultadores & Facilitadores \\
\hline $\begin{array}{l}\text { Coordenação das } \\
\text { ações do programa }\end{array}$ & $\begin{array}{l}\text { - Falta de coordenação entre equipes internas } \\
\text { (apoio habitacional e clínico) (E18,E20,E27 } \\
\text { E33,E57,E60,E66,E68) e com outros prestadores } \\
\text { de serviços necessários para atender as múltiplas } \\
\text { necessidades dos moradores (E6,E58,E18,E24,E27,E } \\
\text { 29,E30,E34,E48,E62,E66) } \\
\text { · Pouca clareza na definição dos papéis e } \\
\text { competências das equipes de apoio habitacional e } \\
\text { clínico (E29,E57) } \\
\text { - Equipe de apoio sobrecarregada pelo acúmulo de } \\
\text { tarefas clínicas e habitacionais (E7,E15,E26,E29) }\end{array}$ & $\begin{array}{l}\text { - Estrutura de governança que define papéis e } \\
\text { responsabilidades, resolve conflitos, articula } \\
\text { rede de serviços e tem boa comunicação } \\
(\text { E15,E20,E29, E49) } \\
\text { - Espaços permanentes de planejamento e } \\
\text { discussão intersetorial com os diferentes agentes } \\
\text { envolvidos (E30) } \\
\text { - Parceria com equipamentos e setores } \\
\text { governamentais para facilitar o acesso a } \\
\text { unidades habitacionais e benefícios de } \\
\text { transferência de renda (E29) } \\
\text { - Parceria com locadores para solucionar } \\
\text { conflitos e favorecer manutenção da moradia } \\
\text { (E29) }\end{array}$ \\
\hline \multicolumn{3}{|c|}{ Processos de execução } \\
\hline Elegibilidade & $\begin{array}{l}\text { - Utilização de critérios de elegibilidade } \\
\text { que desfavorece grupos com menor acesso } \\
\text { a serviços públicos e mais vulneráveis } \\
\text { (E5,E36,E26,E66,E63,E65) } \\
\text { - Expectativa de cumprimento de critérios } \\
\text { para acesso às moradias (tratamento e grau de } \\
\text { funcionalidade) (E34) } \\
\text { - Excesso de discricionariedade da equipe na } \\
\text { elegibilidade dos participantes (E8,E46) } \\
\text { - Restrição de possibilidades de escolha do tipo e } \\
\text { local de moradia aos participantes em decorrência } \\
\text { do grau de necessidade de apoio e histórico de } \\
\text { comportamento (E16,E25) }\end{array}$ & \\
\hline \multicolumn{3}{|c|}{ Estruturação das moradias } \\
\hline $\begin{array}{l}\text { Acesso a imóveis no } \\
\text { mercado privado }\end{array}$ & $\begin{array}{l}\text { · Preconceito e estigma por parte dos proprietários } \\
\text { (E1,E11,E14,E40,E60) } \\
\text { - Exigências específicas de locação, por parte dos } \\
\text { proprietários, para participantes do programa } \\
\text { (verificação de antecedentes criminais, histórico de } \\
\text { crédito) (E17,E37,E38) } \\
\text { - Escassez de moradias acessíveis e em locais de } \\
\text { interesse (E11,E14,E15,E16,E18,E19,E20,E27,E28,E2 } \\
\text { 9,E33,E36,E38,E39,E43,E45,E60,E61,E62,E68) } \\
\text { - Desinteresse do mercado imobiliário (privado e } \\
\text { empresas públicas) em apoiar a intervenção (E42) } \\
\text { - Dificuldades de negociação (tempo e habilidade) } \\
\text { com locadores (E20) } \\
\text { - Falta de mecanismo estruturado para busca de } \\
\text { moradias (E26,E36) }\end{array}$ & \\
\hline $\begin{array}{l}\text { Acesso a imóveis } \\
\text { públicos }\end{array}$ & $\begin{array}{l}\text { - Falta de financiamento para programas de } \\
\text { habitação social (E27) } \\
\text { - Baixa integração do programa com o setor de } \\
\text { habitação pública (E15,E21) } \\
\text { - Limitação na quantidade de habitações sociais } \\
\text { disponíveis (E25) }\end{array}$ & \\
\hline
\end{tabular}


Quadro 2. Fatores que influenciam a implantação do HF sistematizados a partir dos estudos referenciados no Quadro 1.

\begin{tabular}{|c|c|c|}
\hline Fatores & Dificultadores & Facilitadores \\
\hline $\begin{array}{l}\text { Acesso a subsídios } \\
\text { habitacionais }\end{array}$ & $\begin{array}{l}\text { · Subsídios habitacionais inadequados e insuficientes } \\
\text { para custear aluguel, estruturação e manutenção da } \\
\text { moradia (E8,E15,E19,E20,E26,E33,E37,E39,E40,E4 } \\
\text { 2,E48,E60, } \\
\text { E61,E62) } \\
\text { · Experiência limitada de governos locais na } \\
\text { concessão de subsídios habitacionais para } \\
\text { população em situação de rua (E20) } \\
\text { · Dificuldade de divisão dos custos do programa } \\
\text { entre os setores envolvidos na intervenção (E17) }\end{array}$ & \\
\hline $\begin{array}{l}\text { Apoios aos } \\
\text { moradores }\end{array}$ & $\begin{array}{l}\text { - Dificuldade em manter regularidade e } \\
\text { intensidade do apoio clínico aos moradores } \\
\text { (E5,E6,E26,E30,E44,E53) } \\
\text { · Prevalência da atuação na solução de problemas } \\
\text { habitacionais sobre o acompanhamento de } \\
\text { adaptação à moradia (E68) } \\
\cdot \text { Moradias distantes das bases da equipe (E11,E12, } \\
\text { E14,E37,E68) } \\
\cdot \text { Dificuldade de manutenção de equipe } 24 \text { horas } \\
\text { (E11) } \\
\cdot \text { Utilização de práticas controladoras e de alta } \\
\text { exigência (E32,E68) } \\
\cdot \text { Rotatividade de profissionais e gestores (E18,E28) } \\
\text { - Falta de recursos para estruturação completa dos } \\
\text { serviços de apoio (E11) } \\
\text { - Equipes insuficientes para garantir apoio intensivo } \\
\text { aos moradores (E14,E19,E26,E57,E62) } \\
\text { - Dificuldade em efetivar a integração de pares na } \\
\text { equipe (E52) }\end{array}$ & $\begin{array}{l}\text { - Arranjo misto de cuidado (gestão de caso e } \\
\text { tratamento assertivo na comunidade) (E13) } \\
\text { - Utilização de tecnologia à distância para } \\
\text { acompanhamento dos moradores (E13) } \\
\text { - Execução de ações para estabilização } \\
\text { econômica dos participantes (solicitação } \\
\text { de benefícios de transferência de renda e } \\
\text { planejamento financeiro) (E2) } \\
\text { - Trabalhar com vizinhos e proprietários na } \\
\text { prevenção de situações de crise (E2) } \\
\text { - Conhecimento sobre os recursos comunitários } \\
\text { disponíveis e adequados para os beneficiários } \\
\text { (E2) } \\
\text { - Envolvimento de pares no estabelecimento de } \\
\text { uma relação positiva entre morador e equipe } \\
\text { (E10,E29) } \\
\text { - Separação entre equipe de apoio clínico e } \\
\text { habitacional (E41) } \\
\text { - Consideração de aspectos socioeconômicos } \\
\text { (raça, idade, gênero) na escolha dos técnicos de } \\
\text { referência dos moradores (E3) }\end{array}$ \\
\hline
\end{tabular}

Fonte: Autores.

Vencer as barreiras de acesso à moradia e, ao mesmo tempo, garantir apoio intensivo aos moradores constitui o processo central de implantação do HF, missão robusta que exige capacidade técnica, organizacional e de governança. Embora o modelo HF admita que os profissionais das duas áreas que compõem o programa (habitacional e clínica) trabalhem tanto separadamente quanto em uma mesma equipe ${ }^{56}$, diferenças nesses arranjos impactam diretamente a regularidade e intensidade do acompanhamento dos moradores $^{26,63,72-75}$. Obstáculos na adequação do apoio clínico predominam nos diferentes contextos, até mesmo na experiência canadense ${ }^{76}$, considerada a mais consistente ao modelo original HF. Essas limitações podem ajudar a explicar parcialmente as intervenções que não conseguem alcançar melhorias na condição de saúde dos moradores ${ }^{74,77}$. Importa ressaltar que fatores de outras dimen- sões, tais como moradias distantes das bases das equipes e rotatividade de profissionais, também interagem desfavorecendo a qualidade do acompanhamento dos moradores.

A utilização de arranjos mistos de cuidado ${ }^{78}$ que garantam atenção à diversidade e complexidade das necessidades dos moradores, assim como clareza na definição dos papéis, são estratégias que podem diminuir conflitos entre profissionais com diferentes culturas institucionais e promover maior capacidade de implantação. Entretanto, nos casos em que as modalidades de apoio habitacional e clínico são prestadas por diferentes instituições e/ou pertencem a diferentes sistemas, estruturas de governança mais robustas são exigidas para garantir a coordenação dos serviços. Independentemente do arranjo adotado, destaca-se a preocupação de que os responsáveis pelo acompanhamento clínico não sejam 
sobrecarregados com a solução de problemas de estruturação e manutenção das moradias e acabem por adotar uma postura passiva em relação ao investimento na reabilitação psicossocial ${ }^{73,79}$.

Dificuldades específicas para colocar em prática a proposta da Redução de Danos são atribuídas à pouca clareza conceitual e operacional dessa abordagem no âmbito do HF, além da ausência de repertório prático, por parte dos agentes, de competências consideradas essenciais à sua implantação, com destaque para técnicas de entrevista motivacional, planejamento centrado no usuário e manejo clínico de situações de crise $5^{52,63,65,80}$. Por outro lado, a quantidade e a qualidade da interação entre equipe e usuário são apontadas como fator determinante para a construção de relacionamentos positivos que favoreçam a prática da Redução de Danos e do Recovery ${ }^{59,81}$, assim como a oferta de apoio voltado ao enfrentamento da insuficiência econômica dos moradores ${ }^{81}$. Gestores e equipes com valores, atitudes e competências técnicas alinhadas aos princípios do HF, bem como oferta de educação permanente, são relatados como elementos que impulsionam o processo de implantação.

Limitações dos serviços comunitários existentes, que atuam de forma complementar ao HF para atendimento das múltiplas necessidades dos moradores, indicam que as barreiras historicamente enfrentadas por esse público para acesso a bens e serviços básicos (saúde, subsídios de renda mínima, transporte público e meios de comunicação) exigem parcerias e acordos locais mais robustos para a sua superação. $O$ não atendimento dessas necessidades afeta negativamente a implantação, ainda que de outras formas. Por exemplo, a falta de transporte e de telefone dificulta a manutenção de vínculo com familiares e amigos, a participação na vida da cidade e a marcação das visitas de acompanhamento, sendo fatores associados aos sentimentos de isolamento e solidão dos moradores ${ }^{82}$. O mesmo pode ser afirmado para a falta de engajamento e adesão ao programa, a qual pode ser influenciada por experiências negativas em programas similares, falta de transporte para comparecer aos serviços e irregularidade dos serviços ofertados na rede.

A dificuldade de acesso a imóveis e subsídios habitacionais adequados e suficientes, relatada em mais da metade dos estudos analisados e presente em todos os países representados na revisão, aparece como um fator inibidor persistente à materialização do outro componente central da proposta HF: o acesso facilitado à moradia permanente, respeitando a escolha dos partici- pantes. Para além das condições locais do sistema habitacional, que inclui limitações das políticas de habitação social e do mercado imobiliário privado, a indisponibilidade de moradias aparece também associada ao estigma e ao preconceito dos proprietários de imóveis com os postulantes a inquilinos, o que dificulta a estruturação das moradias no tempo e nos locais desejados e implica recorrentes processos de realocação dos participantes em decorrência de conflitos e despejos. Sendo o sistema habitacional um dos maiores desafios não só ao processo de implantação do HF, mas também de desenvolvimento em escala e com sustentabilidade da proposta, o debate sobre sua implantação precisa considerar a crise habitacional vivenciada globalmente e suas particularidades regionais e locais ${ }^{83}$.

Compreende-se que a sistematização dos fatores contextuais nas dimensões propostas e nas categorias dificuldades e facilidades constituiu importante instrumento exploratório para identificar questões relevantes na experiência de implantação do HF, bem como os diferentes níveis em que operam (individual, institucional e social). Entretanto, tal divisão deve ser relativizada, uma vez que os aspectos que constituem o contexto estão relacionados entre si e se configuram como barreiras e/ou facilidades em diferentes graus de importância e intensidade, a depender, por exemplo, do estágio da implantação do HF.

Vale ressaltar que não há consenso na literatura sobre as fronteiras que delimitam o contexto e que, para alguns avaliadores, as dimensões apreciadas neste estudo, por exemplo a concepção da proposta, podem ser consideradas como externas ao contexto e restritas à intervenção ${ }^{60}$. Sobre tal questão, acreditamos que a adoção de uma concepção abrangente de contexto, em nosso caso, mostrou-se vantajosa por resultar em um panorama de fatores que, para além de subsidiar recortes para futuros aprofundamentos de pesquisa, potencialmente permite que realidades de um número maior de contextos sejam contempladas. Por outro lado, os critérios de seleção adotados para esta revisão, especialmente o conjunto de palavras e o tipo de publicação - que se restringiu a estudos empíricos divulgados em revistas científicas - limitaram potencialmente a identificação de um número maior de fatores.

A expressiva quantidade de artigos, quase um terço do total analisado, que trata do mesmo estudo-piloto canadense - At Home/Chez Soi, e a prevalência de um grupo de sete pesquisadores que são autores em mais de um quarto das publicações, são dois aspectos que merecem des- 
taque. Ainda na perspectiva das características dos estudos analisados, importa ressaltar que a maioria dos estudos selecionados não caracteriza suficientemente as condições locais das políticas sociais de saúde, assistência social e habitação nas quais os programas de HF são desenvolvidos, o que reduz a capacidade de analisar quais estratégias podem ser adotadas em circunstâncias particulares de políticas públicas externas ao contexto norte-americano, europeu e australiano. Nesse sentido, a leitura dos fatores aqui sistematizados, em diálogo com uma descrição aprofundada do contexto político e do sistema de proteção social dos países em que o HF foi consolidado, pode contribuir para melhor adequação do modelo às necessidades da América Latina.

Ao se situar o objeto de intervenção do HF, ao menos no Brasil, no campo da política pública (atender à necessidade de proteção social de pessoas em situação de rua que fazem uso de drogas e/ou têm agravos de saúde mental), é fundamental que os entendimentos e propostas formuladas sobre a adaptação do modelo nacionalmente considere os modos de produção de serviços em Saúde e Assistência Social e seus processos de trabalho existentes, assim como as disputas políticas em torno do cuidado público em saúde mental. Dimensões relacionadas ao morar na realidade brasileira, a exemplo do ideário da casa própria e a elevada presença de informalidade nos processos de locação de imóveis, precisam também ser consideradas.

\section{Conclusão}

O presente estudo buscou compreender, por meio de revisão de literatura, os desafios e oportunidades de implantação da intervenção Housing First. Apresentamos um conjunto de fatores relevantes ao processo de implantação desse tipo de intervenção, organizado em quatro dimensões: características da intervenção, contexto de implantação, aspectos institucionais e processo de execução. Verificamos que a indisponibilidade de habitações, a falta de coordenação dos serviços necessários aos moradores e a resistência dos agentes implantadores aos princípios do HF são os principais fatores que dificultam a implantação. Por sua vez, agentes com valores, atitudes e competências convergentes ao modelo e educação permanente são elementos facilitadores.

Consideramos que o estudo da literatura realizado alcançou seu objetivo, fornecendo um conjunto de elementos relevantes na análise, na formulação e no aprimoramento de intervenções que tenham como objetivo garantir o direito à habitação para pessoas com necessidades de apoio em saúde mental, para além de apontar pontos relevantes ao processo de expansão do próprio Housing First. A abrangência das dimensões contextuais consideradas oportunizou uma ampla compreensão do processo de implantação em níveis estruturais e relacionais, que podem ter sua análise aprofundada a partir de recortes e referenciais teóricos delimitados.

Apontamos a relevância da produção de novos conhecimentos que favorecem a integração de intervenções HF aos sistemas de proteção social existentes, processo determinante para sua implantação e sustentabilidade no âmbito da política pública.

\section{Colaboradores}

Os dois autores participaram da concepção, análise e interpretação dos dados, assim como da redação do artigo.

\section{Agradecimentos}

Agradecemos ao Wagner Oda pela contribuição na etapa de seleção dos artigos. 


\section{Referências}

1. Friesinger JG, Topor A, Bøe TD, Larsen IB. Studies regarding supported housing and the built environment for people with mental health problems: A mixed-methods literature review. Health and Place 2019; 57:44-53.

2. Dorvil H, Morin P, Beaulieu A, Robert D. Housing as a social integration factor for people classified as mentally Ill. Hous Stud 2005; 20(3):497-519.

3. Tsemberis S. Housing First: The Pathways Model to End Homelessness for People with Mental Health and Substance Use Disorders. Minnesota: Hazelden; 2015.

4. Tsemberis S, Eisenberg RF. Pathways to housing: Supported housing for street-dwelling homeless individuals with psychiatric disabilities. Prim Care Companion J Clin Psychiatry 2001; 3(1):34-35.

5. Stefancic A, Tsemberis S, Messeri P, Drake R, Goering PAS. The pathways housing first fidelity scale for individuals with psychiatric disabilities. Am J Psychiatr Rehabil 2013; 16(4):240-261.

6. Goering P, Veldhuizen S, Watson A, Adair C, Kopp B, Latimer E, Nelson G, Macnaughton E, Streiner D, Aubry T. National at Home/Chez Soi Final Report. Calgary; 2014 [cited 2020 ago 25]. Available from: www. mentalhealthcommission.ca/English/at-home\#national

7. Busch-Geertsema V. Housing First Europe-Results of a European Social Experimentation Project. Eur J Homelessness 2014; 8(1).

8. Johnson G, Parkinson S, Parsell C, Institute AH and UR, Centre RR. Policy shift or program drift? Implementing Housing First in Australia. AHURI Final Rep [Internet] 2012;184. [cited 2020 ago 25]. Available from: https://www.ahuri.edu.au/research/final-reports/184

9. Padgett D, Henwood BF, Tsemberis SJ. Housing first : ending homelessness, transforming systems, and changing lives. New York: Oxford University; 2016. 228 p.

10. O'Campo P, Stergiopoulos V, Nir P, Levy M, Misir V, Chum A, Arbach B, Nisenbaum R, To MJ, Hwang SW. How did a Housing First intervention improve health and social outcomes among homeless adults with mental illness in Toronto? Two-year outcomes from a randomised trial. BMJ Open 2016; 6(9):e010581.

11. Kirst M, Zerger S, Misir V, Hwang S, Stergiopoulos V. The impact of a Housing First randomized controlled trial on substance use problems among homeless individuals with mental illness. Drug Alcohol Depend 2015; 146(1):24-29.

12. Padgett DK, Stanhope V, Henwood BF, Stefancic A. Substance use outcomes among homeless clients with serious mental illness: Comparing housing first with treatment first programs. Community Ment Health J 2011; 47(2):227-232.

13. Buchanan D, Kee R, Sadowski LS, Garcia D. The health impact of supportive housing for HIV-positive homeless patients: a randomized controlled trial. Am J Public Health 2009; 99(Supl. 3).

14. Gulcur L, Stefancic A, Shinn M, Tsemberis S, Fischer SN. Housing, hospitalization, and cost outcomes for homeless individuals with psychiatric disabilities participating in continuum of care and housing first programmes. J Community Appl Soc Psychol 2003; 13(2):171-186.
15. Rudnitzky F, Evans T. Evaluación Proyecto piloto "Incubar" [Internet]. Montevideo; 2020 [acceso 2020 set 14]. Disponible en: http://dspace.mides.gub.uy:8080/ xmlui/bitstream/handle/123456789/1678/DocumentodetrabajoN63.INCUBARinformeevaluación..pdf?sequence $=3$ \& is Allowed $=y$

16. Garcia L, Kinoshita RT, Maximiano V. Uma perspectiva social para o problema do crack no Brasil: implicações para as políticas públicas. In: Pesquisa Nacional sobre o uso de crack: quem são os usuários de crack elou similares do Brasil? quantos são nas capitais brasileiras? Rio de Janeiro: Fiocruz; 2014.

17. Carvalho AP. Moradia Primeiro no contexto da politica de drogas brasileira: análise da implantação de uma intervenção-piloto de moradia assistida para pessoas em situação de rua [dissertação]. São Paulo: Faculdade de Medicina da Universidade de São Paulo; 2020.

18. Nascimento ACP do, Rosa CM. O paradigma ético-estético-político da clínica na produção de subjetividades: o fazer clínico do Palmas que te acolhe. Polêm!ca 2020; 19(3):122-137.

19. Moreira RS, Mendes APT, Sciammarella AP de O, Amado F. População em situação de rua: direitos humanos, politicas públicas e programas de housing first. Rio de Janeiro: Escola de Direito do Rio de Janeiro da Fundação Getulio Vargas.

20. Brasil. Ministério da Mulher, da Família e dos Direitos Humanos (MMFDH). É possivel Housing First no Brasil?: experiências de moradia para população em situação de rua na Europa e no Brasil. Brasília: MMFDH; 2020.

21. Brasil. Ministério da Mulher, da Família e dos Direitos Humanos (MMFDH). Portaria no. 2.927, de 26 de agosto de 2021. Institui o Projeto Moradia Primeiro no âmbito do Ministério da Mulher, da Família e dos Direitos Humanos. Diário Oficial da União 163 ago 2021; Seção 1.

22. Rog DJ, Marshall T, Dougherty RH, George P, Daniels AS, Ghose SS, et al. Permanent supportive housing: Assessing the evidence. Psychiatr Serv 2014; 65(3):287-294.

23. Goering P, Tsemberis S. Housing First and System/ Community Transformation. In: Community Psychology and Community Mental Health. New York: Oxford University Press; 2014. p. 278-291.

24. Worton SK, Hasford J, Macnaughton E, Nelson G, MacLeod T, Tsemberis S, Stergiopoulos V, Goering P, Aubry T, Distasio J, Richter T. Understanding Systems Change in Early Implementation of Housing First in Canadian Communities: An Examination of Facilitators/Barriers, Training/Technical Assistance, and Points of Leverage. Am J Community Psychol. 2018; 61(1-2):118-130.

25. Damschroder LJ, Aron DC, Keith RE, Kirsh SR, Alexander JA, Lowery JC. Fostering implementation of health services research findings into practice: A consolidated framework for advancing implementation science. Implement Sci 2009; 4(1).

26. Nelson G, Stefancic A, Rae J, Townley G, Tsemberis S, Macnaughton E, et al. Early implementation evaluation of a multi-site housing first intervention for homeless people with mental illness: A mixed methods approach. Eval Program Plann 2014; 43:16-26. 
27. Ouzzani M, Hammady H, Fedorowicz Z, Elmagarmid A. Rayyan-a web and mobile app for systematic reviews. Syst Rev 2016; 5(1):210.

28. Stefancic A, Henwood BF, Melton H, Shin S-M, Lawrence-Gomez R, Tsemberis S, et al. Implementing Housing First in rural areas: Pathways Vermont. Am J Public Health 2013; 103(supl. 2):S206-209.

29. Appel PW, Tsemberis S, Joseph H, Stefancic A, Lambert-Wacey D. Housing first for severely mentally ill homeless methadone patients. J Addict Dis [Internet]. 2012; 31(3):270-277.

30. Benjaminsen L. Policy Review Up-date : Results from the Housing First based Danish Homelessness Strategy. Eur J Homelessness 2013; 7(2):109-131.

31. Barrenger SL, Stanhope V, Atterbury K. Discursive Processes Creating Team Culture and Recovery Orientation among Housing First Providers. Am J Psychiatr Rehabil 2015; 18(3):247-264.

32. Stanhope V. The ties that bind: Using ethnographic methods to understand service engagement. Qual Soc Work 2012; 11(4):412-430

33. Worton SK, Hasford J, Macnaughton E, Nelson G, MacLeod T, Tsemberis S, Stergiopoulos V, Goering P, Aubry T, Distasio J, Richter T. Understanding Systems Change in Early Implementation of Housing First in Canadian Communities: An Examination of Facilitators/Barriers, Training/Technical Assistance, and Points of Leverage. Am J Community Psychol 2018; 61(1-2):118-130.

34. Fleury M-J, Grenier G, Vallée C. Evaluation of the implementation of the Montreal at home/chez soi project. BMC Health Serv Res 2014; 14(1).

35. Kumar N, Plenert E, Hwang SW, O'Campo P, Stergiopoulos V. Sustaining housing first after a successful research demonstration trial: Lessons learned in a large urban center. Psychiatr Serv 2017; 68(7):739-742.

36. Weinzierl C, Wukovitsch F, Novy A. Housing First in Vienna: a socially innovative initiative to foster social cohesion. J Hous Built Environ 2016; 31(3):409-422.

37. Nelson G, Caplan R, MacLeod T, Macnaughton E Cherner R, Aubry T, Méthot C, Latimer E, Piat M, Plenert E, McCullough S, Zell S, Patterson M, Stergiopoulos V, Goering P. What Happens After the Demonstration Phase? The Sustainability of Canada's At Home/ Chez Soi Housing First Programs for Homeless Persons with Mental Illness. Am J Community Psychol 2017; 59(1-2):144-157.

38. Macnaughton E, Nelson G, Worton SK, Tsemberis S, Stergiopoulos V, Aubry T, Hasford J, Distasio J, Goering P. Navigating complex implementation contexts: Overcoming barriers and achieving outcomes in a national initiative to scale out housing first in Canada. Am J Community Psychol 2018; 62(1-2):135-149.

39. Hasford J, Nelson G, Worton SK, Macnaughton E, MacLeod T, Piat M, Tsemberis S, Stergiopoulos V, Distasio J, Aubry T, Goering P. Knowledge translation and implementation of housing first in Canada: A qualitative assessment of capacity building needs for an evidence-based program. Eval Program Plann 2019; 75:1-9.

40. Kertesz SG, Austin EL, Holmes SK, Pollio DE, Schumacher JE, White B, Lukas CV. Making Housing First Happen: Organizational Leadership in VA's Expansion of Permanent Supportive Housing. J Gen Intern Med 2014; 29(4):835-844
41. Austin EL, Pollio DE, Holmes S, Schumacher J, White B, Lukas CV, Kertesz S. VA's expansion of supportive housing: Successes and challenges on the path toward housing first. Psychiatr Serv 2014; 65(5):641-647.

42. Colombo F, Saruis T. Social innovation and local welfare: A comparative case study on housing first in Italy and Sweden. Journal fur Entwicklungspolitik 2017 33:85-111.

43. Nelson G, Worton SK, Macnaughton E, Tsemberis S, MacLeod T, Hasford J, Goering P, Stergiopoulos V, Aubry T, Distasio J. Systems change in the context of an initiative to scale up Housing First in Canada. J Community Psychol. 2019 1; 47(1):7-20.

44. van den Berk-Clark C. The Dilemmas of Frontline Staff Working With the Homeless: Housing First, Discretion, and the Task Environment. Hous Policy Debate 2016; 26(1):105-122.

45. Namian D. Governing homelessness through instruments: a critical perspective on housing first's policy instrumentation. Crit Policy Stud 2019; 14(3):303-318.

46. Anderson-Baron JT, Collins D. Not a "forever model": the curious case of graduation in Housing First. Urban Geogr 2018; 39(4):587-605.

47. Barrenger SL, Stanhope V, Atterbury K. Discursive Processes Creating Team Culture and Recovery Orientation among Housing First Providers. Am J Psychiatr Rehabil 2015; 18(3):247-264.

48. Brothers S, Lin J, Schonberg J, Drew C, Auerswald C. Food insecurity among formerly homeless youth in supportive housing: A social-ecological analysis of a structural intervention. Soc Sci Med 2020; 245:112724.

49. Bullen J, Baldry E. "I waited 12 months": how does a lack of access to housing undermine Housing First? Int J Hous Policy 2019; 19(1):120-130.

50. Jetté J, Aubry T, Cherner R, Ecker J, Yamin S, Bourque J. The implementation of a Housing First intervention in Canadian rural region. Can J Community Ment Heal 2015; 33(4):41-59.

51. Nolin D, Jetté J. Specific features of urban and rural areas: A comparative study on the results of the At Home/Chez Soi project in New Brunswick . Can J Community Ment Heal 2015; 33(4):125-140.

52. Stergiopoulos V, O’Campo P, Gozdzik A, Jeyaratnam J, Corneau S, Sarang A, Hwang SW. Moving from rhetoric to reality: adapting Housing First for homeless individuals with mental illness from ethno-racial groups. BMC Health Serv Res 2012; 12(1):345.

53. Brothers S, Lin J, Schonberg J, Drew C, Auerswald C. Food insecurity among formerly homeless youth in supportive housing: A social-ecological analysis of a structural intervention. Soc Sci Med 2020; 245.

54. Stergiopoulos V, O’Campo P, Gozdzik A, Jeyaratnam J, Corneau S, Sarang A, Hwang SW. Moving from rhetoric to reality: Adapting Housing First for homeless individuals with mental illness from ethno-racial groups. BMC Health Serv Res 2012; 12(1).

55. Kriegel LS, Henwood BF, Gilmer TP. Implementation and Outcomes of Forensic Housing First Programs. Community Ment Health J 2016; 52(1):46-55.

56. Tsemberis S. Housing First: The Pathways Model to end homelessness for people with mental illness and adiction. Minnesota: Hazelden; 2015.

57. Ecker J, Aubry T, Cherner R, Jetté J. Implementation evaluation of a housing first program in a small Canadian City. Can J Community Ment Heal 2014; $33(4): 23-40$ 
58. Nicholls CM, Atherton I. Housing First: Considering components for successful resettlement of homeless people with multiple needs. Hous Stud 2011; 26(5):767-777.

59. Tiderington E, Stanhope V, Henwood BF. A qualitative analysis of case managers' use of harm reduction in practice. J Subst Abuse Treat 2013; 44(1):71-77.

60. Champagne F, Brousselle A, Hartz Z, Contandriopoulos A-P, Denis J-L. A Análise da Implantação. In: Brousselle A, Champagne F, Contandriopoulos A-P, Hartz $\mathrm{Z}$, editors. Avaliação: conceitos e métodos. $2^{\mathrm{a}}$ ed. Rio de Janeiro: Fiocruz; 2011. p. 217-238.

61. Hasford J, Nelson G, Worton SK, Macnaughton E, MacLeod T, Piat M, Tsemberis S, Stergiopoulos V, Distasio J, Aubry T, Goering P. Knowledge translation and implementation of housing first in Canada: A qualitative assessment of capacity building needs for an evidence-based program. Eval Program Plann 2019; 75:1-9.

62. Nelson G, Macnaughton E, Goering P, Dudley M, O'Campo P, Patterson M, Piat M, Prévost N, Strehlau V, Vallée C. Planning a Multi-site, Complex Intervention for Homeless People with Mental Illness: The Relationships Between the National Team and Local Sites in Canada's At Home/Chez Soi Project. Am J Community Psychol 2013; 51(3-4):347-358.

63. Volk JS, Yamin S, Jetté J, Aubry T, Bourque J. The at home / Chez Soi project: Community partners' perspectives on the implementation of housing first in Moncton. Can J Community Ment Heal 2015; 33(4):77-90.

64. Johnsen S, Teixeira L. "Doing it Already?": Stakeholder Perceptions of Housing First in the UK. Int J Hous Policy 2012; 12(2):183-203.

65. Fenwick K, Henwood B, Lengnick-Hall R, Stefancic A, Gilmer T. Exploring Variation in Housing First Implementation: The Role of Fit. Hum Serv Organ Manag Leadersh Gov 2019; 43(5):392-406.

66. Gilmer TP, Ojeda VD, Hiller S, Stefancic A, Tsemberis $S$, Palinkas LA, et al. Variations in full service partnerships and fidelity to the housing first model. Am J Psychiatr Rehabil 2013; 16(4):313-328.

67. Bullen J, Fisher KR. Is Housing First for Mental Health Community Support Possible During a Housing Shortage? Soc Policy Adm 2015; 49(7):928-945.

68. Brodkin EZ, Majmundar M. Administrative exclusion: Organizations and the hidden costs of welfare claiming. J Public Adm Res Theory 2010; 20(4):827848.

69. DeVerteuil G. Homeless Mobility, Institutional Settings, and the New Poverty Management. Environ Plan A Econ Sp 2003; 35(2):361-379.

70. Pires R. Implementando desigualdades: reprodução de desigualdades na implementação de políticas públicas. Rio de Janeiro: IPEA; 2019.

71. Dutra R, Arenari B. Implementação de políticas públicas e inclusão social: a atuação de organizações implementadoras com populações de rua usuárias de drogas e reprodução de desigualdades. Boletim de Análise Político-Institucional 2017; 13(9):29-36.

72. Collins SE, Clifasefi SL, Andrasik MP, Dana EA, Stahl N, Kirouac M, Welbaum C, King M, Malone DK.. Exploring transitions within a project-based housing first setting: Qualitative evaluation and practice implications. J Health Care Poor Underserved 2012; 23(4):1678-1697.
73. Greenberg B, Korb S, Cronon K, Anderson R. Supportive housing best practices in a mid-sized US urban community. Housing, Care Support 2013; 16(1):6-15.

74. Kertesz SG, Austin EL, Holmes SK, DeRussy AJ, Van Deusen Lukas C, Pollio DE. Housing first on a large scale: Fidelity strengths and challenges in the VA's HUD-VASH program. Psychol Serv 2017; 14(2):118128.

75. Henwood BF, Stanhope V, Padgett DK. The role of housing: a comparison of front-line provider views in housing first and traditional programs. Adm Policy Ment Heal Ment Heal Serv Res 2011; 38(2):77-85.

76. Macnaughton E, Stefancic A, Nelson G, Caplan R, Townley G, Aubry T, McCullough S, Patterson M, Stergiopoulos V, Vallée C, Tsemberis S, Fleury MJ, Piat $\mathrm{M}$, Goering P. Implementing Housing First Across Sites and Over Time: Later Fidelity and Implementation Evaluation of a Pan-Canadian Multi-site Housing First Program for Homeless People with Mental Illness. Am J Community Psychol 2015; 55(3-4):279-291.

77. Appel PW, Tsemberis S, Joseph H, Stefancic A, Lambert-Wacey D. Housing first for severely mentally ill homeless methadone patients. J Addict Dis 2012; 31(3):270-277.

78. Stefancic A, Henwood BF, Melton H, Shin SM, Lawrence-Gomez R, Tsemberis S. Implementing Housing First in rural areas: Pathways Vermont. $\mathrm{Am} \mathrm{J} \mathrm{Pu-}$ blic Health 2013; 103(Supl. 2):S206-209.

79. Tiderington E. "We Always Think You're Here Permanently": The Paradox of "Permanent" Housing and Other Barriers to Recovery-Oriented Practice in Supportive Housing Services. Adm Policy Ment Heal Ment Heal Serv Res 201:103-114.

80. Collins SE, Malone DK, Larimer ME. Motivation to change and treatment attendance as predictors of alcohol-use outcomes among project-based Housing First residents. Addictive Behaviors 2012; 37:931-939.

81. Andvig ES, Sælør KT, Ogundipe E. Harm reduction in a Norwegian housing first project: a qualitative study of the treatment providers' practice. Adv Dual Diagn 2018; 11(1):4-15.

82. Macnaughton E, Stefancic A, Nelson G, Caplan R, Townley G, Aubry T, McCullough S, Patterson M, Stergiopoulos V, Vallée C, Tsemberis S, Fleury MJ, Piat M, Goering P. Implementing Housing First Across Sites and Over Time: Later Fidelity and Implementation Evaluation of a Pan-Canadian Multi-site Housing First Program for Homeless People with Mental Illness. Am J Community Psychol 2015; 55(3-4):279-291.

83. Molina I, Czischke D, Rolnik R. Housing policy issues in contemporary South America: an introduction. Intern J Housing Policy 2019; 19:277-287.

Artigo apresentado em 27/10/2020

Aprovado em 13/10/2021

Versão final apresentada em 15/10/2021

Editores-chefes: Romeu Gomes, Antônio Augusto Moura da Silva 